\title{
Origin and evolution of deep plasmaspheric notches
}

\author{
D. L. Gallagher \\ NASA Marshall Space Flight Center, Huntsville, Alabama, USA \\ M. L. Adrian \\ NASA Goddard Space Flight Center, Greenbelt, Maryland, USA
}

M. W. Liemohn

Space Physics Research Laboratory, University of Michigan, Ann Arbor, Michigan, USA

Received 17 November 2004; revised 29 April 2005; accepted 6 June 2005; published 1 September 2005.

[1] Deep plasmaspheric notches can extend over more than $2 R_{E}$ in radial distance and 3 hours MLT in the magnetic equatorial plane, as observed by the extreme ultraviolet (EUV) imager on the IMAGE mission. They are among the largest evacuated features in the exterior plasmaspheric boundary. They can last for days and exhibit a variety of shapes. It appears that weak convection and limited erosion precedes notch formation at the westward, near-Earth edge of the convection plume. Eighteen clear notch events were found and analyzed in 2000. Among these events, notches were found to drift as slowly as $44 \%$ of corotation. In only one case was a notch found to drift at the corotation rate within measurement error. On average, these notches drift at about $21.5 \mathrm{~h} \mathrm{~d}^{-1}$ or $90 \%$ of the corotational rate. Notches sometimes exhibit an interior structure that appears as an extended prominence of dense plasma, which forms a W- or M-like feature in IMAGE/EUV images, depending on viewing perspective. Initial modeling suggests that notches and notch prominences may be caused in part by intense small-scale potential structures that result from the localized injection of ring current plasma. Plasma filling rates during recovery are examined in three $\mathrm{L}$ shell ranges from $\mathrm{L}=2$ to $\mathrm{L}=3.5$ with rates ranging from 5 to $140 \mathrm{~cm}^{-3} \mathrm{~d}^{-1}$. Plasma loss during a minor substorm is found to extend to surprisingly low $\mathrm{L}$ shell with rates ranging from 100 to $130 \mathrm{~cm}^{-3} \mathrm{~d}^{-1}$ across the L shells examined.

Citation: Gallagher, D. L., M. L. Adrian, and M. W. Liemohn (2005), Origin and evolution of deep plasmaspheric notches, J. Geophys. Res., 110, A09201, doi:10.1029/2004JA010906.

\section{Introduction}

[2] The plasmasphere, a relatively dense toroidal region of cold plasma surrounding the Earth, has been studied for many years [see Lemaire and Gringauz, 1998] and is thought to play an important role in energetic particle scattering and the transport of energy in the inner magnetosphere [e.g., Fok et al., 1993; Liemohn et al., 2000; Khazanov et al., 2003]. Density structures on a variety of scale sizes have been found in plasmaspheric plasma [Carpenter et al., 2002; Sandel et al., 2003]. The outer boundary of the plasmasphere, referred to as the plasmapause or plasmasphere boundary layer, is often characterized by an abrupt one to two orders of magnitude drop in plasma density. During extended geomagnetically quiet conditions this region may lack a sharp boundary and instead exhibit a density that gradually falls to trough levels. The most significant azimuthal plasmaspheric structure is the plume, which extends sunward in afternoon and evening local times. The dominant mechanism for plume formation is the global

Copyright 2005 by the American Geophysical Union. 0148-0227/05/2004JA010906 cross-tail electric field induced by the solar wind streaming through the Earth's outer magnetic field [Nishida, 1966; Grebowsky, 1970]. Recently, it has become clear that the details of plume structure are also dependent on more localized electric fields [Foster et al., 2002; Goldstein et al., 2004a, 2004b; Liemohn et al., 2004].

[3] Notches [Sandel et al., 2003] represent one of the largest density structures in the plasmasphere, along with the plasmasphere itself, the plasmaspheric plume, and plasmaspheric channels. Notches may contribute to in situ observations previously described as density cavities inside the plasmasphere [Carpenter et al., 2002]. This possibility was demonstrated by Green et al. [2002], where corotation of a plasmaspheric notch in combination with orbital satellite motion was found able to reproduce the in situ measurement properties of an interior density cavity. Notches have recently been found to play an important role in the generation of kilometric continuum radiation [Green et al., 2002, 2004], known for many years to be a pervasively observed plasma wave in the outer magnetosphere low-density cavity.

[4] Notches are characterized by deep density depletions that extend mostly radially inward to $\mathrm{L}=2$ or less. The sizes 


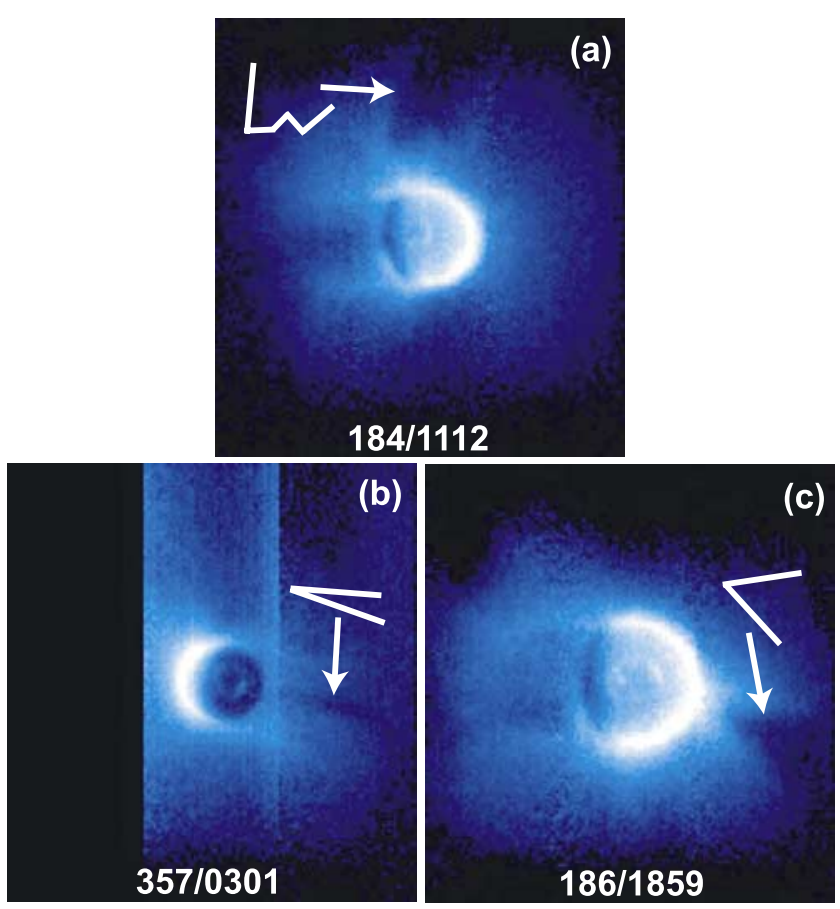

Figure 1. $(\mathrm{a}-\mathrm{c})$ Examples of plasmaspheric notches are shown in EUV observations from 2000. Each panel is shown with the day of year and universal time of the observation. They are found to extend over two or more L shells and to be as narrow as 0.1 MLT and as broad as several hours MLT. Notches can be simple gaps in density or contain structure such as the interior prominence shown in Figure 1a.

in local time range from very narrow $(\sim 0.1$ hours MLT) to very broad ( $\sim 3$ hours MLT). Notch densities observed by extreme ultraviolet (EUV) are found to be a factor of 510 below the adjacent notch walls, although interior notch densities often fall to the EUV noise level so that notch depletions may be much deeper. Figure 1 shows three examples of plasmaspheric notches. Each panel is a 10-min integrated image acquired by the EUV camera on the IMAGE mission. The camera observes $30.4 \mathrm{~nm}$ sunlight resonantly scattered by $\mathrm{He}^{+}$ions in the plasmasphere [Sandel et al., 2000]. Counts increase logarithmically from dark blue to bright blue and then white. The Earth is in the center of each image and the bright arc close to the Earth is ionospheric glow on the sunward side. Figure 1a shows a structured notch toward the top of the image near dusk local time. Notches sometimes include a central prominence of enhanced plasma density that can be somewhat broad as in this case or exceptionally narrow and extending radially across two or more L shells within the notch. Figure $1 \mathrm{~b}$ shows one of the more narrow notches. This notch extends from about $\mathrm{L}=1.6$ to $\mathrm{L}=5.2$ before it can no longer be seen by the EUV instrument. Figure 1c shows a notch of similar simple structure but much more broad in local time.

[5] All plasmaspheric densities and related quantities presented in this work are for $\mathrm{He}^{+}$ions, not total electron density. An estimate of the helium to hydrogen ratio can be obtained from the statistical study by Craven et al. [1997]. A discussion of the correspondence between IMAGE EUV observations of helium ions and total electron density can be found in the work by Goldstein et al. [2003]. In this second study, a good correspondence was found between steep density gradients observed in EUV images of $\mathrm{He}+$ and those inferred from upper hybrid resonant emissions measured by the IMAGE Radio Plasma Imager [Reinisch et al., 2000]. It is for that reason the $\mathrm{He}^{+}$density gradients observed for notches by the EUV instrument are assumed here to also correspond to that for total electron density.

[6] The present work explores the origin of these deep, large density cavities in the outer plasmasphere and their evolution. Only clear, distinct notches observed during 2000 are examined here. The appearance of especially narrow (in local time) notches is more subject to viewing geometry and difficult to follow in time. Notchlike features that are broad in local time, but shallow in L shell, may be related to the notches explored here or may be related to another morphological feature referred to as crenulations. Crenulations are irregular features in plasmapause L shell whose origins are unclear, although they appear to bear similarity to shoulder features associated with overshielding and undershielding in the inner magnetosphere [Goldstein et al., 2002; Spasojević et al., 2003]. It is found that notches appear to share their origin with low-density channels, which are formed during recovery at the base of the plasmaspheric plume in the dusk region. Long-lived notches present the opportunity to follow their refilling and motion across a wide range of L shells. In one notch, refilling is found to be consistent with previous early time refilling, but responsive to a brief increase in magnetic activity. Notches are also found to routinely drift eastward at a rate below corotation [Sandel et al., 2003] and often at the same rate across a wide range of inner L shells.

\section{Origin of a Notch}

\subsection{Observational Evidence}

[7] Figure 2 shows the plasmasphere near the start of recovery from a period of enhanced convection. Each panel shows a plume in the dusk region that will soon drift eastward in the corotation direction. While otherwise similar, the first two events on 31 May 2000 and 30 June 2000 precede the formation of a notch. The third event on 10 June 2000 results in an extended low-density channel. These three event periods are shown again a short time later in Figure 3. A much more clear difference has developed in the plasma distribution between the first two (Figures 3a and $3 b$ ) and the third event (Figure 3c) as shown from left to right in Figure 3. The first two events develop into radial notch structures, while the third event forms a low-density, azimuthal channel inside a wrapped plume. Of the few notches so far observed during formation, all appear to originate at the westward edge and base of the convection plume upon recovery from enhanced storm-time convection. The entrainment and wrapping of the convection plume during storm-time recovery was first proposed by Grebowsky [1970] and subsequently discussed in many studies [see, e.g., Carpenter et al., 1992; Sandel et al., 2001; Spasojević et al., 2003]. The process results in an azimuthally extended and radially narrow region of low density inside a similarly extended region of enhanced density that was once the sunward extended plume. 

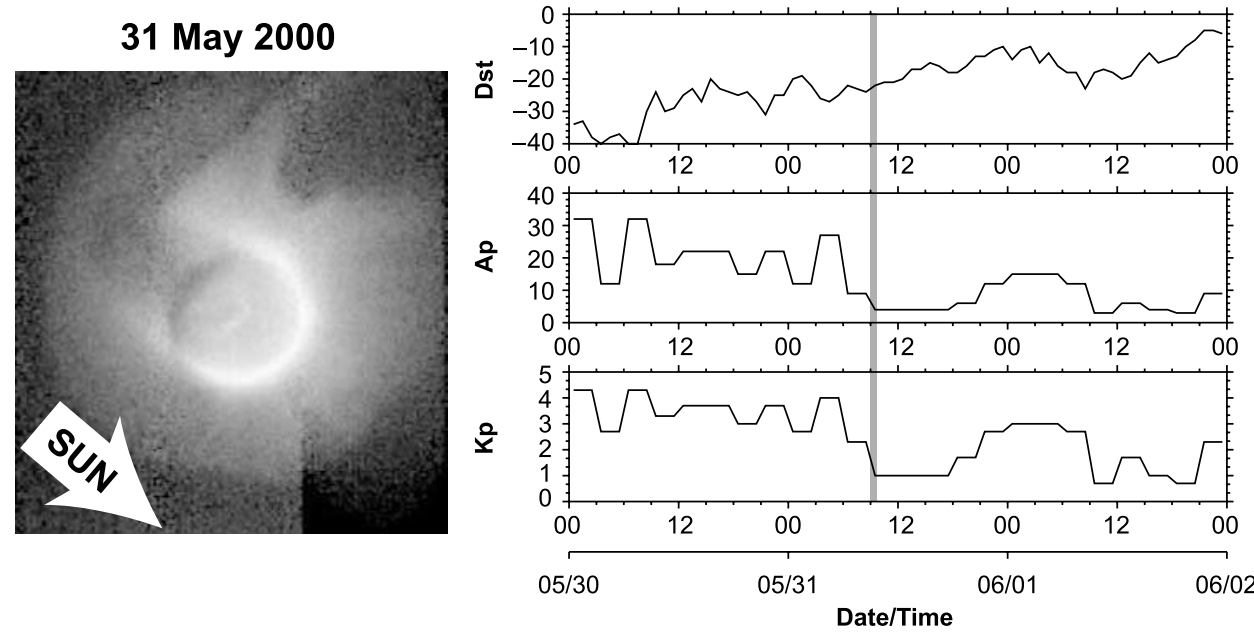

30 June 2000
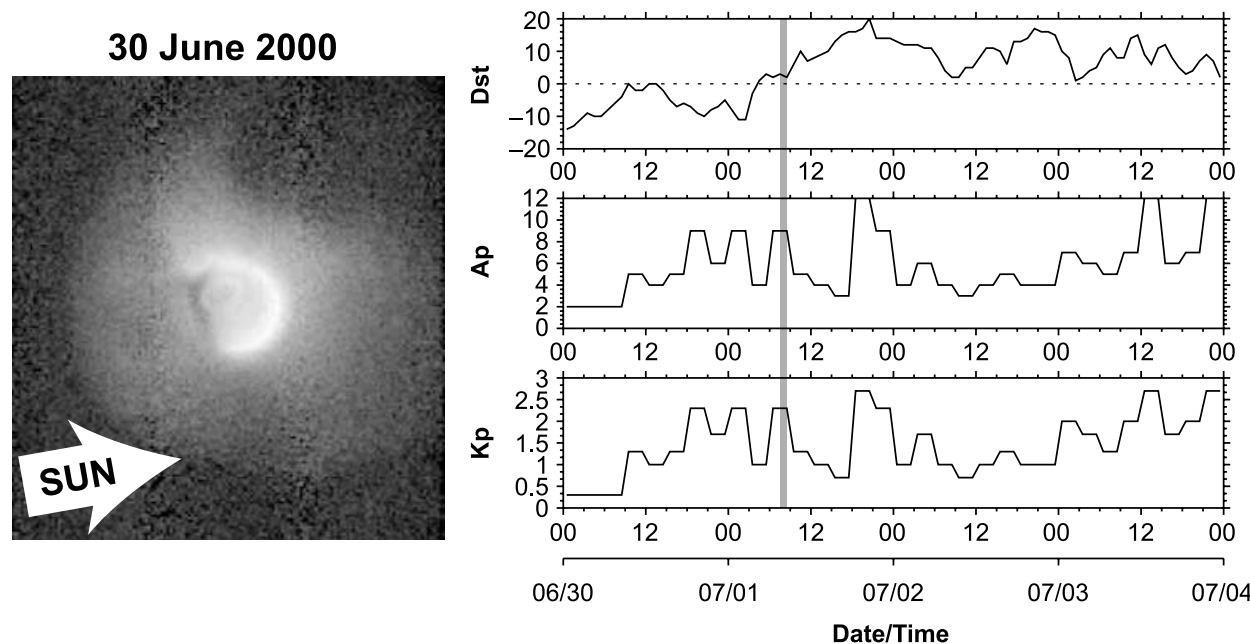

10 June 2001
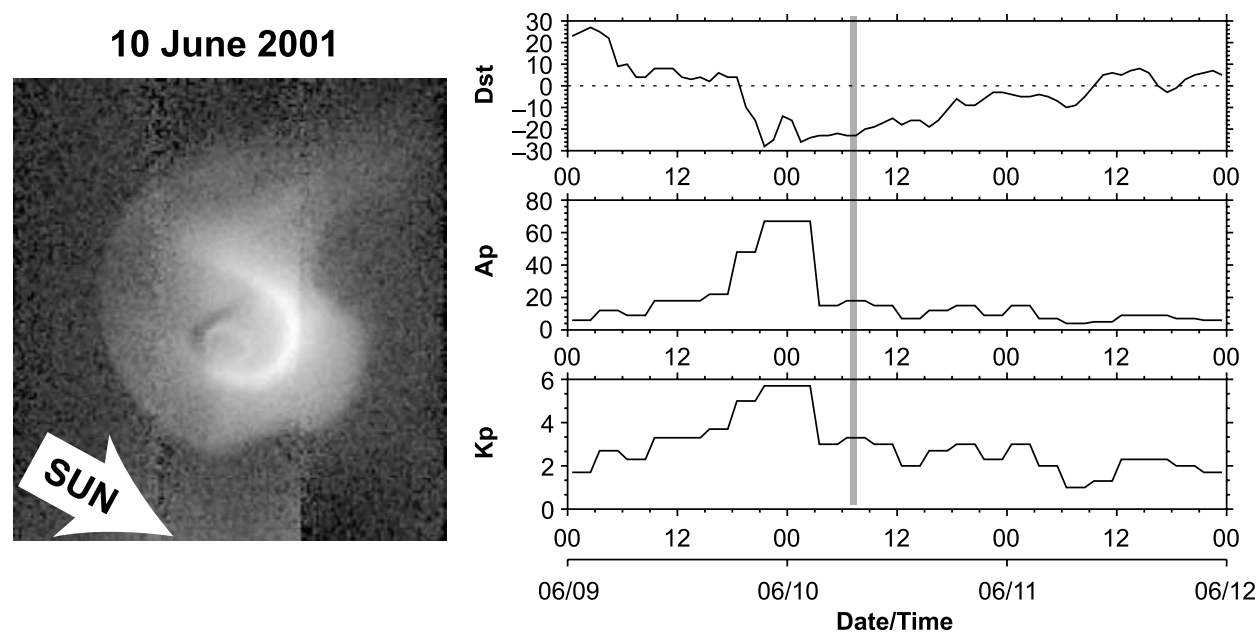

Figure 2. Storm-time recovery of the plasmaspheric plume on three different days. Two of the days on 31 May 2000 and 30 June 2000 result in the formation of a notch. The day of 10 June 2000 results in the formation of a low-density channel.

[8] From EUV observations during 2000, notches appear to originate during storm-time recovery in the same region as low-density channels. Observationally, the distinction between notch and low-density channel formation appears to be due to the size and/or plasma content of the storm-time plume and correspondingly to the degree of plasmaspheric erosion. In order to illustrate these distinctions, two periods of notch formation (Figures $2 \mathrm{a}$ and $2 \mathrm{~b}$ and Figures $3 \mathrm{a}$ and $3 \mathrm{~b}$ ) are compared to a period of low-density channel formation (Figures $2 \mathrm{c}$ and $3 \mathrm{c}$ ). The two notch 
31 May 2000

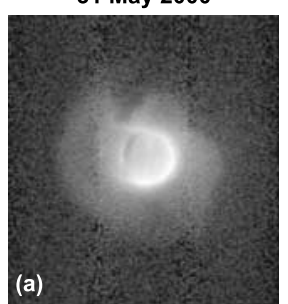

30 June 2000

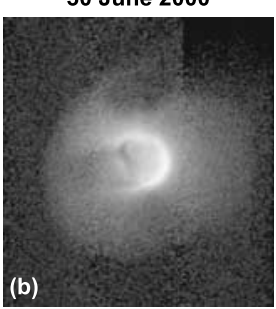

10 June 2000

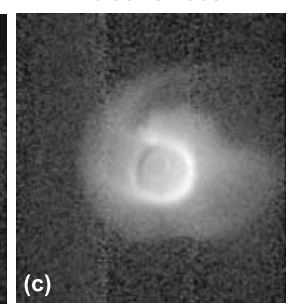

Figure 3. EUV images of the plasmasphere showing notches and a channel formed later in storm-time recovery on the days shown in Figure 2. ( $a$ and b) Notches early in the formation process. (c) Shows a channel still forming.

events are characterized by a thin plume and limited erosion of the plasmasphere. Much more of the outer plasmasphere was eroded in the channel event. With the aid of the cartoon presented in Figure 4, we illustrate the observed differences in notch and channel formation. In Figure 4, time proceeds from left to right with notch formation shown across the top of Figure 4 and channel formation across the bottom. The upper sequence illustrates that limited plasmaspheric erosion and smaller erosion plume leads to the formation of a plasmaspheric notch, while more extensive plasmaspheric erosion and plume are associated with the development in recovery of a long, thin plume draped across the plasmasphere forming a low-density channel.

[9] Careful examination of the events shown in Figures 1a and $3 \mathrm{~b}$ reveal the presence of a thin wispy remnant of the convection plume overdraping the notch density cavity. While not always visible in EUV images, we hypothesize that a remnant plume often remains, at least for a short period of time after the formation of a notch. The details of electric fields and other thermal plasma drivers that control the distinction between notch and channel formation are left to subsequent analysis. It should be noted that in some cases extended low-density, channel-like structures are observed to reorient into radial low-density notches. Likewise, EUV has also observed features that were likely W-shaped notches (see below) entrained within the eastward extent of low-density channels. Also, while the residual azimuthally draped plume in notch events quickly disappears from EUV images, an observationally similar, but slower disappearance is observed in the wrapped plumes that result in low-density channels [Grebowsky, 1970; Chen and Wolf, 1972; Adrian et al., 2001].

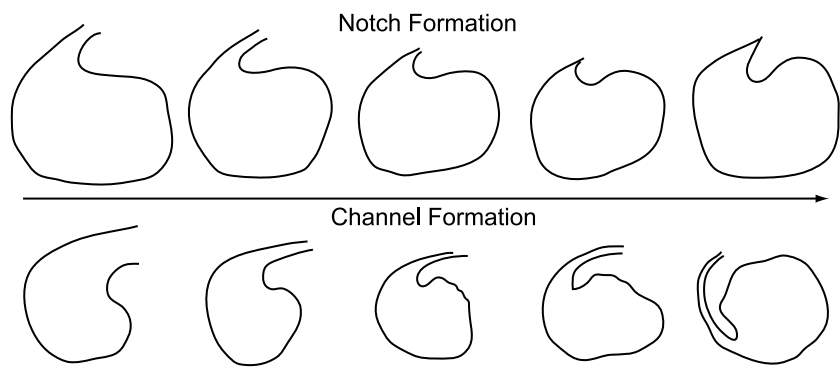

Figure 4. A cartoon summarizing the observed morphology for plasmaspheric notch and channel formation. Time proceeds from left to right, with notch formation along the top and channel formation along the bottom.
[10] In order to more easily examine plasmaspheric features and variations in plasma content over time, subsequently shown EUV images are projected into the dipole magnetic equatorial plane and counts are transformed into pseudodensity, where modeled variations in solar irradiance at $30.4 \mathrm{~nm}$ and the most dominant systematic influences of image intensity across the field of view are removed. This analysis is discussed in Appendix A.

[11] A notch sometimes includes an interior azimuthally narrow radial density enhancement, or prominence. An example of such a prominence is shown in Figure 1a. In this example, the prominence is nearly as broad as the notch near the Earth and rapidly narrows in azimuth with increasing $\mathrm{L}$ shell such that it forms something like a "W" in enhanced density. Although not visible in this rendering of the event, prominences can sometimes be seen to extend across the entire radial length of the notch and with a very narrow azimuthal extent $\left(\sim 0.1 R_{E}\right)$.

[12] An interior prominence can be seen to form just after notch formation during the event on 24 June 2000, which is shown in Figure 5. Pseudodensity images mapped into L shell versus MLT are shown at 30 min intervals over a period of 2 hours. Only the 5-hour MLT region centered on the notch is shown in each image. In the left most image the notch has recently formed just westward of the recovering convection plume. The notch walls become more radial and distinct as the feature evolves leaving an enhanced prominence near the notch center. While a definitive explanation for the formation of a notch prominence is not yet available, a candidate mechanism can be suggested.

\subsection{Computational Explanation}

[13] A possible mechanism for the formation of a plasmaspheric notch and a prominence within a notch is intense, small-scale electric field structures in the inner magnetosphere. This is best described by considering a few numerical results. Figure 6 shows magnetic equatorial plane plots of plasmaspheric density from the dynamic global core plasma model (DGCPM) [Ober et al., 1997]. Results from
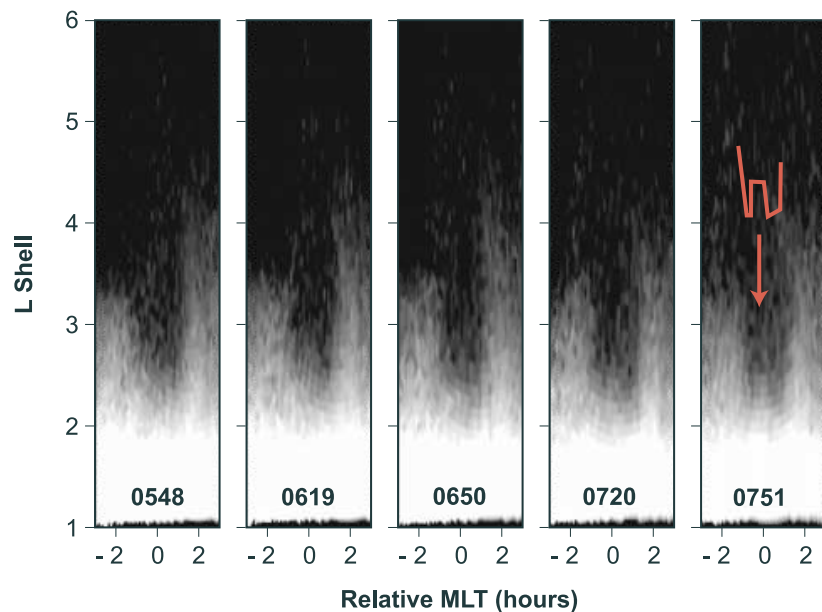

Figure 5. A prominence forming inside the notch that developed on 24 June 2000. Universal time is shown toward the bottom of each panel, where EUV intensities have been projected into the dipole magnetic equatorial plane and expressed in pseudodensity. 

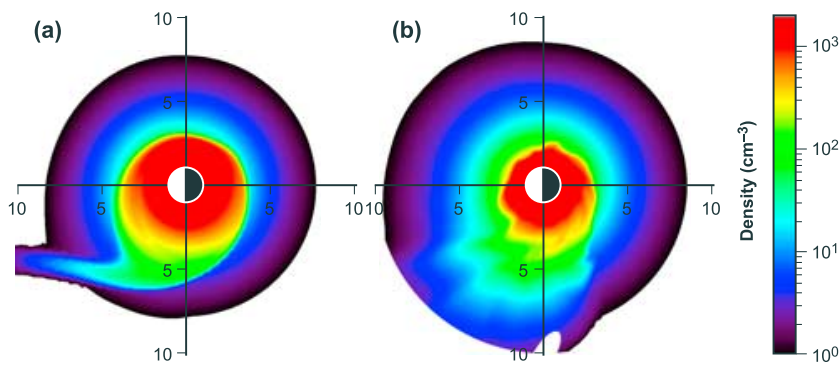

Figure 6. Equatorial plane plasmaspheric density plots from the DGCPM for the recovery phase of the 17 April 2002 magnetic storm. The two results use (a) a smoothly varying two-cell convection pattern and (b) a convection pattern generated self-consistently from a concurrent ring current simulation. The view is from over the north pole with noon to the left and distances are given in $R_{E}$.

two simulations are shown, one with a prescribed convection electric field description (Figure 6a) and one with an electric field that is self-consistently calculated from the inner magnetospheric field-aligned currents produced by a simulation of energetic ring current plasma (Figure 6b). Both plots are for the same instant during the recovery phase of the 17 April 2002 magnetic storm. Details of the computational setup for these results are discussed by Liemohn et al. [2004].

[14] Figure 6 a yields the typical smoothly varying teardrop plasmapause with convection drainage plume morphology first modeled by Grebowsky [1970]. Figure 6b exhibits several features that resemble the plasmaspheric notches seen in the IMAGE EUV data. At $\sim 15$ LT there is a small indentation in the plasmapause; near $18 \mathrm{LT}$ is a V-shaped depletion corresponding to the wrap of the drainage plume around the storm-time plasmapause; and at 21 LT is yet another depleted notch within the wrapped up plume structure. These low-density regions are not seen in Figure 6a. The initial conditions and the ionospheric source and loss terms for these two simulations are exactly the same; the only difference is the convection electric field specification. The field yielding the plasmasphere in Figure $6 \mathrm{a}$ is a well-behaved two-cell convection pattern, while the field used to produce the plasmasphere in Figure $6 \mathrm{~b}$ is distorted from the standard two-cell scenario by a rather large potential well near midnight and several small-scale, transient electric potential vortices superposed on the main convection pattern.

[15] The electric potential vortices appear as a consequence of the injection of hot plasma sheet ions into the ring current region. Figure 7 gives a schematic illustration of this process. Influxes of particles create localized pressure peaks that must have field-aligned currents on the eastward and westward ends of the peak to close the asymmetric ring current loop. These field-aligned currents produce wells and peaks (eastward and westward ends, respectively) in the ionospheric electric potential pattern, which in turn can be mapped back out to the magnetosphere and alter the plasma flow through near-Earth space. The plasma motion is a clockwise flow around the electric potential peak (westward end) and a counterclockwise flow around the well (eastward end). The net result is a radially outward flow between the peak and the well and inward flows to the outside (in the azimuthal direction) of the well-peak pair. As the hot ion pressure peak drifts around the dusk side of the inner magnetosphere, the associated potential structure will also move westward through the region, eventually dissipating on the dayside.

[16] The cold, plasmaspheric particles, which have essentially no magnetic drift, are therefore a tracer of the time history of the convective drift pattern. The formation of a notch in the plasmapause greatly depends on the local time location and extent of the hot ion injection. During a storm, there are many successive injections from the plasma sheet into the inner magnetosphere, which could cause numerous indentations and undulations in the plasmapause. Of course, this ring current-induced deformation of the plasmapause is highly dependent on the ionospheric conductance, which regulates the strength of the resulting ionospheric potential pattern through Ohm's law. This may contribute to why notches appear during certain disturbed times and not others. A more rigorous computational analysis of notch formation is planned for the near future.

\section{Notch Refilling}

[17] The conversion of EUV observations to pseudodensity, mapping into the magnetic equator, and tracking of features makes it relatively easy to follow changes in notch content as it evolves. Notches are often found to persist as an identifiable structure for an extended period of time [Sandel et al., 2003]. Figure 8 shows a sequence of images that extends across the entire lifetime of a notch that formed on 31 May 2000, during recovery from a moderate storm $\left(\mathrm{Kp}_{\max }=4+\right)$. The first image was taken at 0859UT and is shown in Figure 8 as 0000 hours. The following images to the right show the time in hours and minutes relative to this first image time. The notch remains a prominent feature for nearly 32 hours before refilling. In order to estimate the filling rate, weighted averages of pseudodensity in a region

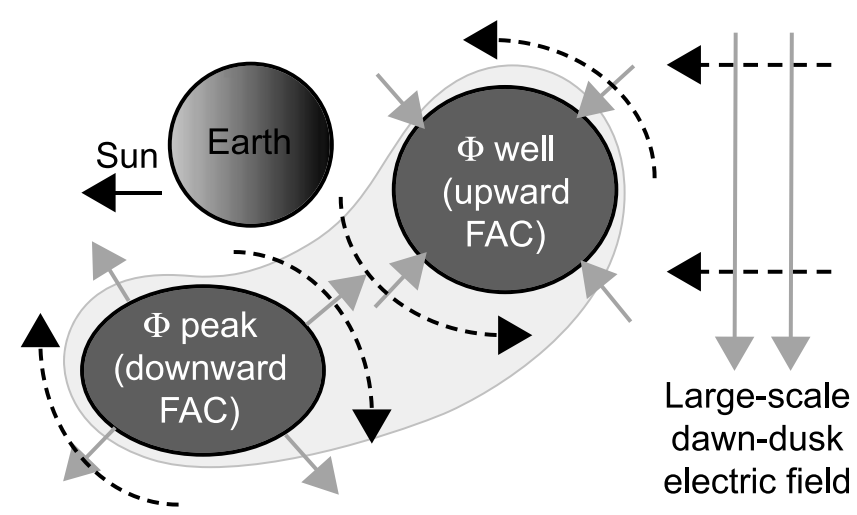

Figure 7. A sketch for the relationship between the partial ring current, the field-aligned closure currents, and the electric fields in the inner magnetosphere. The lightly shaded region represents a near-Earth pressure peak in the evening sector, with corresponding field-aligned currents at each end (dark shaded regions within the light one). Electric field vectors are shown by the shaded arrows, and the corresponding drift flows are drawn with solid, dashed-line arrows. 


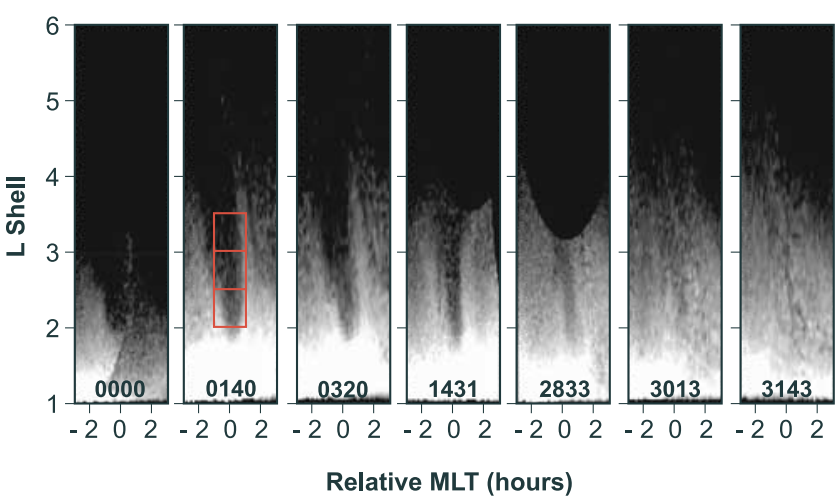

Figure 8. Notch formed on 31 May 2000 sampled throughout its life. Times shown are relative to the time of the first image of 0831 UT on this day. It can be seen that the notch extends across roughly $2 R_{E}$ in radial distance and 2 hours MLT in EUV images. A weighted average of plasmaspheric pseudodensity is computed for each image across the three regions illustrated by the box outlines shown on the image at 0140 hours.

centered on the notch are computed and then followed in time. The regions for which pseudodensity is averaged are shown in the second panel of Figure 8 by the red boxes that extend in 0.5 intervals from $\mathrm{L}=2$ to $\mathrm{L}=3.5$ and for one hour on either side of the notch center. The weighting function is the area represented by each image element in the areas considered.

[18] The weighted density as a function of time for this notch is shown in Figure 9 and is found to vary considerably during the three IMAGE orbital passes when the notch can be followed. During the first 5 hours and from the innermost range to the outermost, refilling rates are $5.5 \pm$ $21 \mathrm{~cm}^{-3} \mathrm{~d}^{-1}, 46.8 \pm 3.8 \mathrm{~cm}^{-3} \mathrm{~d}^{-1}$, and $49.4 \pm 9.9 \mathrm{~cm}^{-3} \mathrm{~d}^{-1}$, respectively. As can be seen, the densities for the innermost L shell range are essentially constant, while densities in the outer two $\mathrm{L}$ shell ranges rise at similar rates. During this first orbital pass the notch is centered between 18.1 hours and 21.8 hours MLT. During the second pass the notch is located between 7.1 hours and 8.6 hours MLT. While in this postdawn region the notch experiences a modest increase in activity $(\mathrm{Kp}=3)$ around $0000 \mathrm{UT}$ on 1 June 2000. As a consequence, plasma is lost throughout the notch during this period of modest activity with loss rates of $-101 \pm 45.5 \mathrm{~cm}^{-3} \mathrm{~d}^{-1},-128 \pm 29 \mathrm{~cm}^{-3} \mathrm{~d}^{-1}$, and $-128 \pm 29 \mathrm{~cm}^{-3} \mathrm{~d}^{-1}$, respectively. Refilling is found again during the third orbital pass when the notch is centered from 19.9 hours to 23.3 hours MLT. Refilling rates are $75 \pm$ $18 \mathrm{~cm}^{-3} \mathrm{~d}^{-1}, 139 \pm 14 \mathrm{~cm}^{-3} \mathrm{~d}^{-1}$, and $80 \pm 11 \mathrm{~cm}^{-3} \mathrm{~d}^{-1}$. Unlike at the beginning of the observational period, there is clear refilling across the whole $\mathrm{L}$ shell range during this time period. As is expected, the averaged densities across all times are highest for $L$ shells in the range $2.0 \leq L \leq 2.5$ and lowest in the range $3.0 \leq L \leq 3.5$.

[19] The refilling rates derived from the EUV notch observations are similar to the refilling rate of $\sim 80 \mathrm{~cm}^{-3} \mathrm{~d}^{-1}$ for $\mathrm{L}=4.5$ obtained by Carpenter et al. [1993]. Park [1973] obtained a somewhat lower value of $30-40 \mathrm{~cm}^{-3} \mathrm{~d}^{-1}$ for $\mathrm{L}=$ 4.5 , while Chappell [1974] obtained a rate of $\sim 50 \mathrm{~cm}^{-3} \mathrm{~d}^{-1}$ for $\mathrm{L}=4$. At geosynchronous orbit Lawrence et al. [1999] measured an early time refilling at a rate of $\sim 0.6-12 \mathrm{~cm}^{-3}$ $\mathrm{d}^{-1}$ and a later time refilling rate of $10-50 \mathrm{~cm}^{-3} \mathrm{~d}^{-1}$. By using a dipole magnetic field, an estimate of $\sim 50 \times$ difference in flux tube volume and hence refilling rate might be anticipated between the Lawrence et al. [1999] observations and those presented here. On the basis of that estimate, our early recovery time refilling rates are comparable to those found at geosynchronous orbit [see also Décréau, 1983, 1986; Higel and Wu, 1984; Song et al., 1988].

\section{Notch Drift}

[20] In addition to following the refilling of plasmaspheric flux tubes, the cross-field drifts of 18 notches have been tracked. The MLT location of notch centers near $\mathrm{L}=2.5$ are first approximated manually. These locations are then used as initial conditions for a least squares Gaussian function fit to the azimuthal notch profile. The Gaussianderived notch centers are then followed in time. Table 1 shows the azimuthal drift rates as hours per day. A feature drifting with the Earth's rotation would be shown at a rate of 24 hours per day. Only one notch was observed to corotate with the Earth; the one observed on 28 May 2000. Most of the remaining notches drift at a rate between $85 \%$ and $97 \%$ of corotation. Two notches observed from 21-23 December 2000 were found to drift considerably slower at $44 \%$ and $74 \%$ the corotation rate, respectively. Figure 10 shows an example of a notch that could be followed for three days. The symbols show the MLT location of the (Gaussianderived) notch center during the observational period. The solid line is a linear fit to the notch centers, which gives a drift rate that is $91 \%$ of the corotation rate. Were the notch to strictly corotate with the Earth, its location would follow the dotted line. While this notch was followed for a longer time than usual, the ability to approximate its subcorotation with a linear function is typical. Even when minor varia-

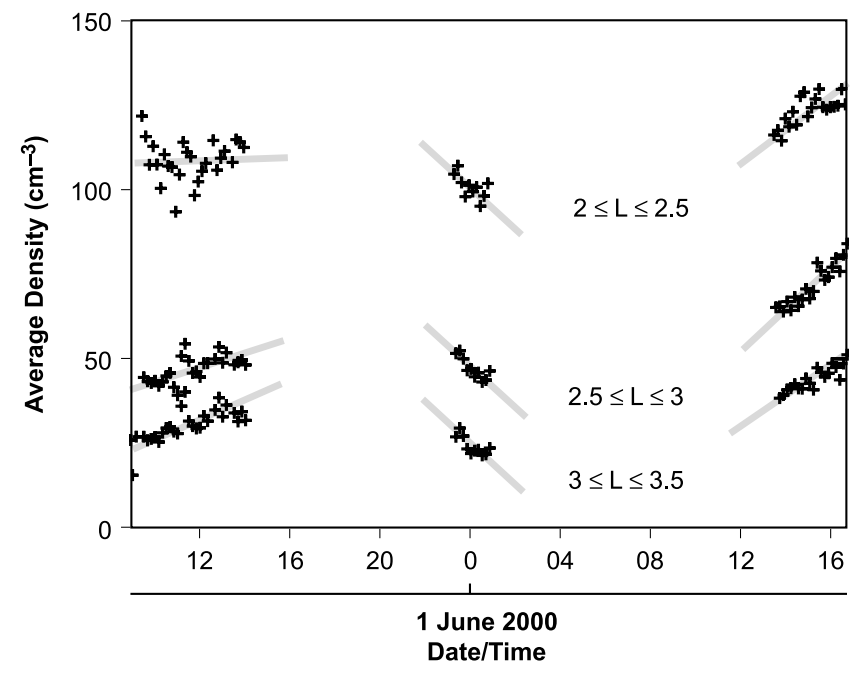

Figure 9. Weighted average density as a function of time plotted for the event shown in Figure 8. Weighted averages are computed for the $0.5 \mathrm{~L}$ and 2 hour bins that follow the notch center. Shaded bars correspond to linear fits to each segment of average densities, indicating an initial gradual increase, then minor storm-related decrease, and then more pronounced increase at the end of the event. 
Table 1. Plasmaspheric Notch and Ionospheric Drifts

\begin{tabular}{lcc}
\hline Dates in 2000 & IMAGE/EUV $\left(\mathrm{h} \mathrm{d}^{-1}\right)$ & DMSP/IDM $\left(\mathrm{h} \mathrm{d}^{-1}\right)$ \\
\hline 27 May & $22.2 \pm 0.12$ & $21.2 \pm 1.5$ \\
28 May & $24.0 \pm 0.09$ & $\ldots$ \\
31 May to 1 June & $22.4 \pm 0.08$ & $22.5 \pm 3.4$ \\
14-15 June & $20.4 \pm 0.07$ & $24.3 \pm 3.7$ \\
16-17 June & $22.6 \pm 0.08$ & $24.7 \pm 1.4$ \\
24-25 June & $23.2 \pm 0.06$ & $21.0 \pm 0.5$ \\
30 June & $21.6 \pm 0.07$ & $20.5 \pm 20.5$ \\
1 July & $22.5 \pm 0.13$ & $24.1 \pm 1.8$ \\
3-5 July & $22.6 \pm 0.11$ & $16.6 \pm 1.0$ \\
6-9 July & $22.2 \pm 0.06$ & $\ldots$ \\
12-14 July & $21.9 \pm 0.64$ & $22.6 \pm 1.1$ \\
27-28 July & $20.7 \pm 0.11$ & $22.0 \pm 1.3$ \\
30-31 July & $22.8 \pm 0.05$ & $\ldots$ \\
6 August & $19.5 \pm 0.76$ & $\ldots$ \\
6-10 August & $21.9 \pm 0.07$ & $\ldots$ \\
21-22 December & $10.5 \pm 0.08$ & $\ldots$ \\
22-23 December & $17.8 \pm 0.16$ & $21.8 \pm 1.1$ \\
28-31 December & $22.1 \pm 0.10$ & .0 \\
\hline
\end{tabular}

tions away from a linear fit are found, an overall linear drift with time dominates the behavior of a notch. In addition, notches often substantially maintain their spatial shape during their lifetime. Some of the apparent changes in notch structure may be attributed to changes in observing geometry. A closer examination of the evolution of notch shape is left for subsequent study.

[21] Burch et al. [2004] have proposed that subcorotation of the plasmasphere is driven by subcorotation of the ionosphere. They go on to propose that the ionospheric disturbance dynamo drives ionospheric motion relative to corotation as described by Blanc and Richmond [1980]. Burch et al. [2004] tested their hypothesis by comparing ionospheric drift measurements from the DMSP spacecrafts against EUV derived plasmaspheric drift. The present notch

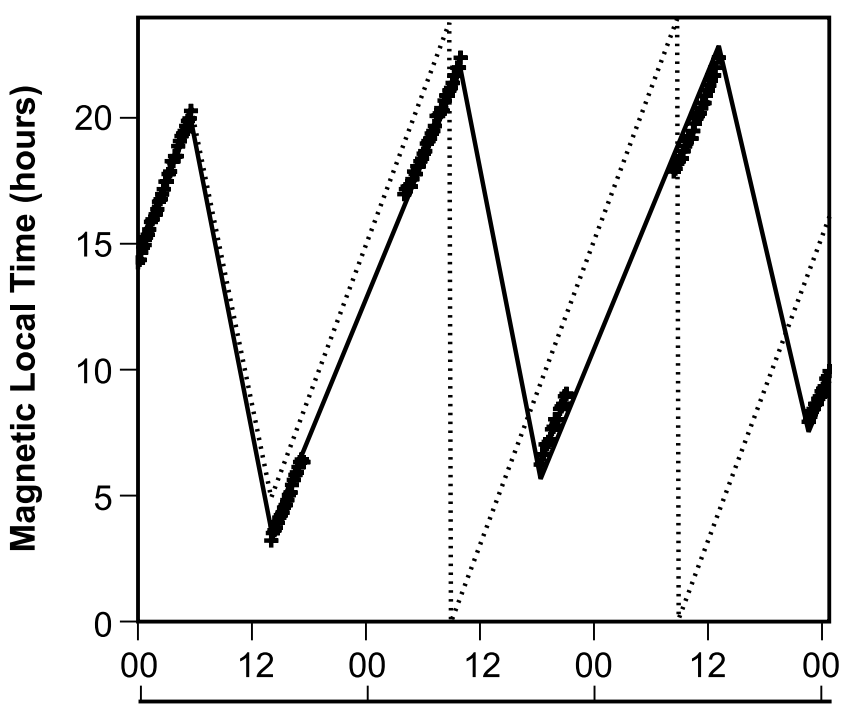

\section{August 2000}

9 August 2000

Figure 10. A notch center tracked across 3 days in August 2000 and shown as pluses. The solid line is a linear fit to the azimuthal notch drift at $2.19 \pm 0.07 \mathrm{~h} \mathrm{~d}^{-1}$. The dotted line indicates corotational drift. measurements offer another opportunity to test this hypothesis. Ion Drift Meter (IDM) observations from the DMSP spacecraft numbers F12, F13, and F15 have been used to obtain average drift for time periods when a DMSP orbit passes within the $L$ shell range $2 \leq L \leq 3$ and within 2 hours MLT of the notch location as observed by IMAGE EUV. IDM drift measurements during these conjunctions are averaged and included in Table 1. The relative correspondence between derived notch drift rates and ionospheric drift rates can be seen in Figure 11. Within the margin of error, most average ionospheric drifts are consistent with notch drift.

[22] Notably, that is not true for all cases. One such case, for 24-25 June 2000, is highlighted in Figure 12. Here, individual IDM drift measurements are compared to the derived linear notch drift. IDM drift measurements are shown in Figure 12 (top) where each symbol is an ionospheric drift measurement. Each grouping of symbols results from multiple IDM measurements during one DMSP pass near the notch location. The dotted line corresponds to corotation. The solid red line is the linear drift of the notch derived from EUV. Ionospheric drift rates are systematically slower that that of the notch. In Figure 12 (bottom) the relative magnetic longitude of the notch, in hours, is plotted through the observational period. The solid line is the linear fit. The dotted line indicates strict corotation. The plasmaspheric drift indicated by EUV is easily more than one standard deviation from the average ionospheric drift from IDM. That is also true for the notch observed on $3-$ 5 July 2000.

[23] A possible explanation for the differences found between IDM and EUV drifts might be due to magnetic local time differences in the observations. As stated above, the IDM measurements included in ionospheric drift averages are within 2 hours MLT of the IMAGE spacecraft

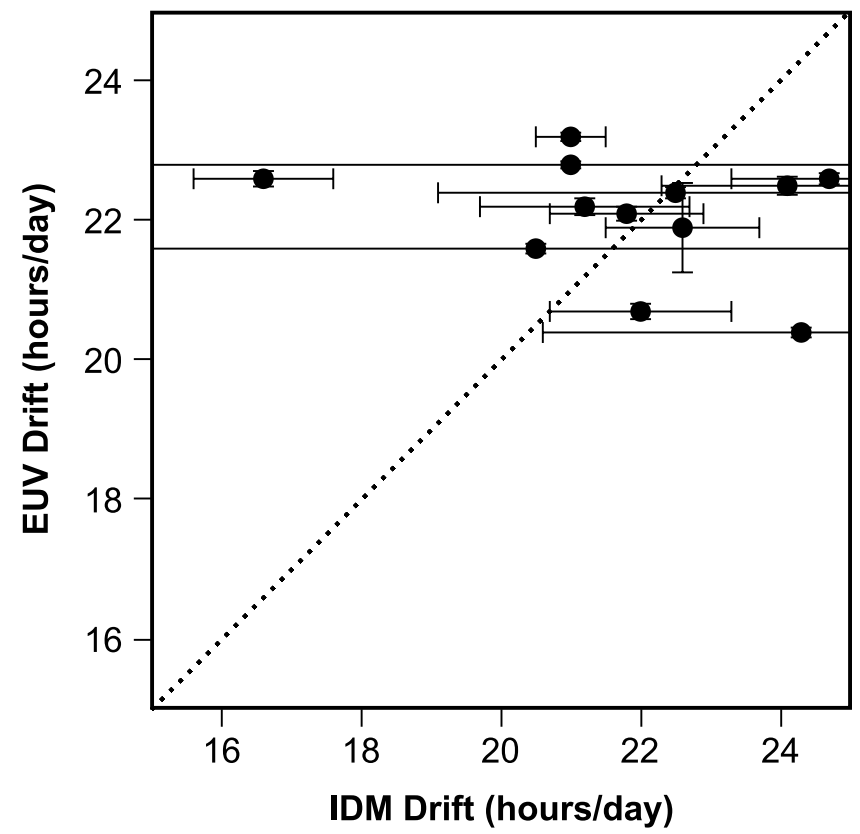

Figure 11. A scatterplot for EUV-derived plasmaspheric and IDM-derived ionospheric drifts. Error bars are drawn one standard deviation to either side of the average drifts. 

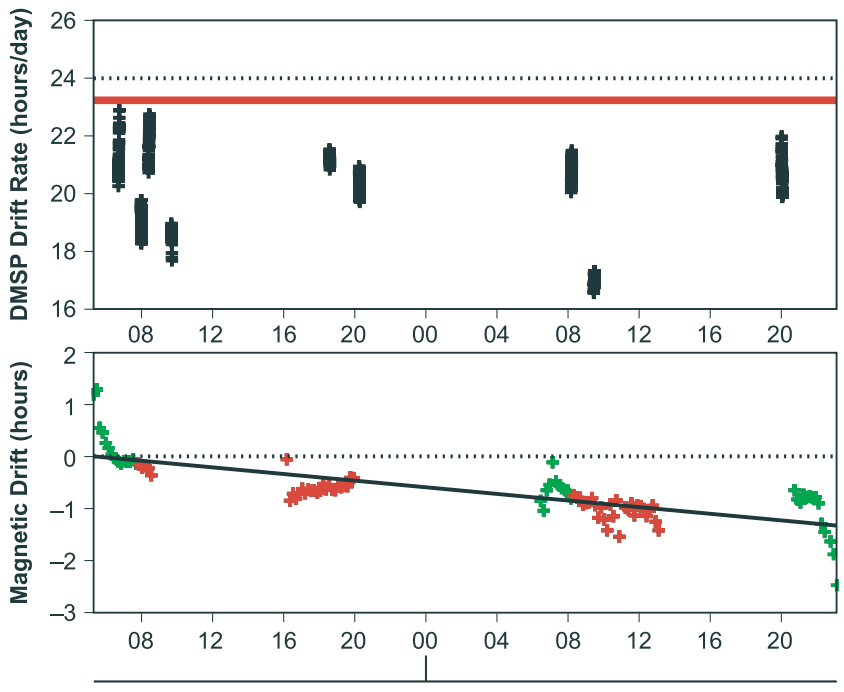

25 June 2000

Figure 12. (top) Drift rate derived from the IDM on DMSP satellites as a function of time on 24-25 June 2000. Each collection of symbols represents a separate DMSP pass through the vicinity of the notch in space and time. The dotted line corresponds to strict corotation. The red line is the notch drift derived from the EUV instrument. (bottom) Magnetic drift of the notch relative to the initial time of the observation period. The solid line is a least squares fit to the notch location. The dotted line represents strict corotation. Green symbols are for dayside notch locations, while red symbols are for the nightside.

location. Should there exist variation in ionospheric drift on smaller azimuthal scales, then the different drifts found here do not contradict the ionospheric slippage proposed by Burch et al. [2004]. In an attempt to test that possibility, IDM measurements have been resampled for the June 2000 notch with much more strict MLT criteria for correspondence. In this case, DMSP was required to be within 0.5 hours MLT of the EUV-observed notch at the time of the observation in addition to being between $\mathrm{L}=2$ and $\mathrm{L}=3$. Three of the nine DMSP orbital passes shown in Figure 12 are within 0.5 hours MLT of the notch center. The drifts measured by IDM are still easily lower in drift rate than that obtained from the EUV instrument. The IDM measurements were obtained from the University of Texas at Dallas and include quality flags that are intended to reflect the likelihood that IDM measurements accurately reflect ionospheric drifts. Only those measurements assigned the highest-quality flag were included in this study. While the other DMSP average drifts are statistically consistent with the notch drifts derived here, there remains considerable scatter in DMSP drift values.

[24] As mentioned above, it is not uncommon to find the observed short-term notch location to drift somewhat slower and faster than the long-term trend, but identification of a long-term trend appears well justified. Green and red colors are used in Figure 12 to represent notch location in and out of sunlight, respectively. This annotation was applied to the analysis of all events for the purpose of revealing whether the day/night changes in ionospheric conductivity might contribute to a diurnally varying slippage of the notch location relative to the long-term drift. No such dependence was found. Observational geometry was also considered as a possible source of apparent short-term shifts in notch position. In a format similar to Figure 12, the spacecraft angular location relative to the plane of the notch and the rate of spacecraft motion transverse to the plane of the notch were examined along side the short-term shifts in notch position relative to the long-term trend. The idea here is that an observing location out of the plane of a notch might result in a systematic error in locating the notch in magnetic longitude. Similarly, the rate of motion of the observing location toward or away from the notch might lead to a systematic increase or decrease of the apparent rate of motion of a notch. Again, no such systematic correlation could be found to explain these short-term shifts.

[25] Another explanation for the notch (and, in general, plasmaspheric) subcorotation is the dawn-dusk asymmetry of the electric potential pattern [e.g., Lu et al., 1989; Boonsiriseth et al., 2001; Ridley et al., 2004]. For instance, Lu et al. [1989] found that the potential difference from the pole to the equator along the dusk meridian is typically 1.5 times larger than the potential difference along the dawn meridian. Ridley et al. [2004] explained this asymmetry as a result of the Hall conductance gradient at the terminators. In a three-dimensional Ohm's law [Amm, 1996], there is a term proportional to the product of the meridional (north-south) electric field and the azimuthal (east-west) gradient of the Hall conductance. The net effect of this term is to reduce the magnitude of the dawnside potential peak and increase the size of the duskside potential well (compare Ridley et al. [2004, Plates 4 and 6]). For nominal, nonstormtime conductance values, Ridley et al. [2004] found that the duskside potential minimum was $23 \%$ larger than the dawnside potential maximum. A similar term in Ohm's law that includes the azimuthal gradient of the Pedersen conductance yields an asymmetry of only $1 \%$ or $2 \%$ in the opposite direction (that is, a bigger dawnside peak). The potential pattern asymmetry results in a stronger sunward convection on the duskside of the magnetosphere than on the dawnside, which is a difference not accounted for in standard two-cell convection patterns. The symmetric convection scenario leads to subcorotative flow on the duskside and supercorotative flow on the dawnside, with no net influence of convection on the drift period along closed drift paths (in steady state). However, the convective asymmetry creates a larger decrease on the duskside and a smaller increase on the dawnside, resulting in drift periods longer than 24 hours (that is, subcorotation). Liemohn et al. [2004] show that the inner magnetospheric component of the dawn-dusk asymmetry varies with storm phase (in the self-consistent electric field results), indicating that the subcorotation effect is modulated by field-aligned current and conductance variations. The disturbance dynamo discussed by Burch et al. [2004] also causes subcorotative drift periods. Both effects will cause the same net westward flow in the DMSP drift data. A determination of the relative contribution of these two effects is beyond the scope of this paper and intended for a later study.

\section{Discussion and Conclusions}

[26] Notches are one of the remarkable large-scale structural features of the plasmasphere, only recognized after 
flight of the remote sensing EUV instrument on the IMAGE spacecraft. Notches are characterized by nearly radial cavities in plasma density that often extend over $2 R_{E}$ in the magnetic equator and from a tenth of an hour to two hours or more in MLT. The notch density cavity can extend inward to $\mathrm{L}=1.6$ or less and is sometimes found to be transiently "capped" at the outer plasmaspheric boundary by a thin, residual plasmaspheric plume. Excavation of plasmaspheric densities to such low altitudes is remarkable by itself and cannot be explained by global convection as evidenced in the work of Carpenter and Anderson [1992]. We speculate here that mesoscale electric field structures, possibly resulting from localized storm-time injection, are necessary to create these spatially deep features. Densities in a notch can be at least a factor of 5-10 lower than the adjacent notch walls. Notches appear to form following weak periods of enhanced convection on the westward edge and base of the plasmaspheric plume. They can maintain their form for several days during quite conditions. Notches are also capable of loosing plasma while maintaining shape during subsequent weak periods of increased magnetic activity.

[27] A central enhanced density prominence was found in about $22 \%$ of the notches identified in 2000 . On 24 June 2000 , a prominence was observed to form soon after or with formation of a notch. Evidence is presented here that this prominence may be indicative of a spatially localized injection of plasma sheet ions and the formation of enhanced mesoscale regions of opposite electric potential. These small-scale potential enhancements appear capable of locally drawing plasma out of the interior high-density region into the low-density notch through modification of the $\mathbf{E} \times \mathbf{B}$ convection pattern. As discussed above, such small-scale potential structures may also result in localized inward convection of plasma, possibly leading to the low L shell penetration of notch features. Although not presented, notch prominences do not necessarily stay centered in the notch even though the notch itself maintains its general shape. In two cases, interior prominences are observed to drift westward relative to the notch, later merging with the notch interior wall.

[28] The large, low-density region of a notch lends itself to the study of plasmaspheric refilling and examination of cross-field drift over extended periods of time. One refilling period was examined closely. During the event on 31 May and 1 June 2000, early changes in average density in the innermost $\mathrm{L}$ shell range are somewhat mixed with little overall refilling. The middle and outer $\mathrm{L}$ shell range, however, show a similar rate of refilling in the range of $47-49 \mathrm{~cm}^{-3} \mathrm{~d}^{-1}$. Refilling between 2 and 3 times this rate is observed during the third IMAGE orbital pass in all $\mathrm{L}$ shell ranges. This last period of early time refilling is observed when the densities are higher than that present for the first orbital pass observing period. These refilling ranges are consistent with those reported by Lawrence et al. [1999] and others as discussed above.

[29] Of some interest is the loss of plasma in the innermost $\mathrm{L}$ shell range $(2.0 \leq L \leq 2.5)$ during the modest increase in magnetic activity to $\mathrm{Kp}=3$ near the start of 1 June 2000. On the basis of Carpenter and Anderson [1992], the plasmapause might be expected to erode inward to $\mathrm{L}=3$ for this level of activity. However, essentially the same rate of plasma loss is seen inside that L shell as is seen outside. Convective plasma loss cannot explain what is found inside of $\mathrm{L}=3$. Carpenter [1962] is the first to report this type of plasma loss inside a storm-time plasmapause. Drainage into the ionosphere is another avenue for plasma loss, which was first proposed by Park [1973]. This dayside plasma loss may also relate to the nightside density loss inside the storm-time eroded plasmapause found by Carpenter [1995]. Successful explanation of this low L shell plasmaspheric erosion will also need to operate near dawn as found here.

[30] As a plasmaspheric feature extended in $\mathrm{L}$ shell, notches directly support the examination of convective drift across a significant range of $\mathrm{L}$ shells. Table 1 summarizes our findings for 18 notches observed during 2000. Only one of these notches was found to drift with the rotation of the Earth. In two cases, the plasmasphere drifted much slower, as slow as $44 \%$ of corotation. We find that a notch most often drifts eastward at a rate of $85-97 \%$ of corotation. The only conclusion we can reach is that the plasmasphere usually lags corotational motion; however, the slippage is often not large. Burch et al. [2004] has suggested that westward ionospheric drift is responsible for slowing the corotational motion of the plasmasphere. Just as in this cited study, we have obtained IDM ionospheric drift measurements for 12 of 18 notches. One standard deviation error estimates for these ionospheric drifts suggest that most are consistent with our notch drift rates.

[31] For two of the notches studied here, the IDM drift rates are significantly slower than found using EUV. The implication is that the explanation for subcorotational drift of the plasmasphere may be more complex than currently thought. In this regard, we note the works of $L u$ et al. [1989], Boonsiriseth et al. [2001], Ridley et al. [2004], and Liemohn et al. [2004] may provide an additional explanation for subcorotational drift. These works collectively suggest that Hall conductance gradients at the terminators cause a dawn-dusk electric potential asymmetry, yielding a net subcorotational plasmaspheric drift that is storm phase-dependent.

\section{Appendix A}

[32] Observed EUV instrument counts are converted to column density [Sandel, private communication] using the following equation (A1):

$$
N={ }^{a \cdot 1.89 \times 10^{19}} / F,
$$

where $N$ is the $\mathrm{He}+$ column abundance in $\mathrm{cm}^{-2}, a$ is the EUV signal in counts/pixel for a 10-min integration, and $F$ is the solar irradiance at $30.4 \mathrm{~nm}$ in units of photons . $\mathrm{cm}^{-2} \cdot \mathrm{s}^{-1}$. Solar irradiance is obtained from the SOLAR2000 irradiance model [Tobiska, 2004].

[33] Column integrated density is converted to pseudodensity by dividing by an estimate of the distance along the line of sight that contributes most to the image intensity at each location in the field of view. Because of rapidly falling densities in the plasmasphere with increasing $\mathrm{L}$ shell, the innermost regions penetrated by a given line of sight will contribute most to the observed $30.4 \mathrm{~nm}$ intensity [Sandel et $a l ., 2003]$. The EUV imager spatial resolution in the 


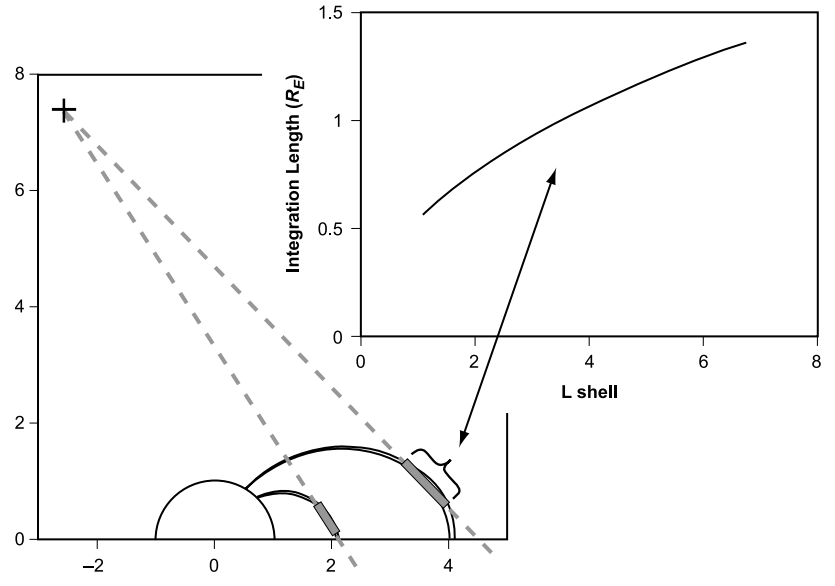

Figure A1. Lowest L shells contribute most to observed $30.4 \mathrm{~nm}$ light intensity because of strongly falling densities in the plasmasphere. The line of sight distance through the lowest L shells effectively increases as a high-latitude imager looks further from the Earth. The sketch in the bottom left outlines the effect. The plot in the top right displays a typical change in effective integration length with imaged L shell. Up to a factor of about 3 change in the contribution of a given density to observed $30.4 \mathrm{~nm}$ intensity can result in an imager's field of view.

equatorial plane while observing from apogee is about 0.1 $R_{E}$, therefore that distance along the line of sight when passing within $0.1 R_{E}$ of the innermost $\mathrm{L}$ shell reached is divided into the column integrated density for each position in the EUV field of view. A sketch describing this influence is shown in Figure A1 along with a typical example of how this effective integration length changes with line of sight below a high-latitude observing location.

[34] By choosing observing periods when the IMAGE spacecraft is at high latitude ( $>60$ degrees magnetic latitude) and relatively far from perigee ( $>4 R_{E}$ geocentric distance), the regions dominating the intensities observed in EUV images are relatively close to the magnetic equator. Relatively little change in density within the plasmasphere is anticipated along magnetic field lines near the equator [Gallagher et al., 2000; Reinisch et al., 2004], therefore EUV images are next mapped to the dipole magnetic equator [Sandel et al., 2003]. Dipole coordinates are used, since distortions from dipole are small close to the Earth during the periods of quiet geomagnetic activity examined in this study.

[35] The quantitative accuracy of the pseudodensity calculation has been tested by comparison to a known density. The dynamic global core plasma model (DGCPM) [Ober et al., 1997] was used to simulate a storm-time recovery period on 10 June 2001 . The simulation resulted in a nightside, narrow plume and otherwise normal plasmasphere with a relatively sharp plasmapause boundary. This equatorial distribution of plasma was then used to define densities along the magnetic field. This run of DGCPM does not include an ionosphere and is limited to modeling $\mathrm{L}$ shells beyond 2; therefore no model ionosphere is included in this test. Simulated EUV images through this modeled environment were then produced for satellite positions at a distance of $8 R_{E}$ and at magnetic latitudes of $60^{\circ}, 70^{\circ}, 80^{\circ}$, and $90^{\circ}$. Pseudodensities where then computed for each image and compared to the original density distribution. Accuracy improved notably with increasing latitude, especially in the region viewed on the far side of the Earth. Most derived densities are within about $50 \%$ of the original, but vary by as much as a factor of nearly 10 in localized regions. Density at a sharp plasmapause tended to be underestimated. Densities just inside the plasmaspheric plume, in a low-density channel overdraped by the plume, are overestimated. Naturally, densities in the Earth's shadow are underestimated by the pseudodensity calculation.

[36] Acknowledgments. The IMAGE Mission through the NASA Office of Space Science has supported this research. The DMSP drift meter data were provided by the Center for Space Sciences at the University of Texas at Dallas through their website at http://cindispace.utdallas.edu/ DMSP/. The authors particularly acknowledge access to the IMAGE/ EUV observations through the University of Arizona website at http:// euv.lpl.arizona.edu/euv/ and much useful insight into the instrument data gained by discussions with Bill Sandel and Terry Forrester. The authors acknowledge important improvements to the manuscript that resulted from the thorough and insightful comments of the reviewers.

[37] Arthur Richmond thanks the reviewers for their assistance in evaluating this manuscript.

\section{References}

Adrian, M. L., D. L. Gallagher, J. L. Green, and B. R. Sandel (2001), The large-scale plasmaspheric density trough associated with the 24 May 2000 geomagnetic storm: IMAGE EUV observations and Global Core Plasma Modeling, Eos Trans. AGU, 82, S352.

Amm, O. (1996), Comment on "A three-dimensional, iterative mapping procedure for the implementation of an ionosphere-magnetosphere anisotropic Ohm's law boundary condition in global magnetohydrodynamic simulations," Ann. Geophys., 14, 773.

Blanc, M., and A. D. Richmond (1980), The ionospheric disturbance dynamo, J. Geophys. Res., 85, 1669.

Boonsiriseth, A., R. M. Thorne, G. Lu, V. K. Jordanova, M. F. Thomsen, D. M. Ober, and A. J. Ridley (2001), A semiempirical equatorial mapping of AMIE convection electric potentials (MACEP) for the January 10, 1997, magnetic storm, J. Geophys. Res., 106, 12,903.

Burch, J. L., J. Goldstein, and B. R. Sandel (2004), Cause of plasmasphere corotation lag, Geophys. Res. Lett., 31, L05802, doi:10.1029/ 2003GL019164.

Carpenter, D. L. (1962), The magnetosphere during magnetic storms, a whistler analysis, Tech. Rep. 12, Radiosci. Lab., Stanford Univ., Stanford, Calif., June.

Carpenter, D. L. (1995), Earth's plasmasphere awaits rediscovery, Eos Trans. $A G U, 76(9), 89$.

Carpenter, D. L., and R. R. Anderson (1992), An ISEE/Whistler model of equatorial electron density in the magnetosphere, J. Geophys. Res., 97, 1097.

Carpenter, D. L., A. J. Smith, B. L. Giles, C. R. Chappell, and P. M. E. Decreau (1992), A case study of plasma in the dusk sector associated with enhanced magnetospheric convection, J. Geophys. Res., 97, 1157. Carpenter, D. L., B. L. Giles, C. R. Chappell, P. M. E. Décréau, R. R. Anderson, A. M. Persoon, A. J. Smith, Y. Corcuff, and P. Canu (1993), Plasmasphere dynamics in the duskside bulge region: A new look at an old topic, J. Geophys. Res., 98, 19,243.

Carpenter, D. L., M. A. Spasojević, T. F. Bell, U. S. Inan, B. W. Reinisch, I. A. Galkin, R. F. Benson, J. L. Green, S. F. Fung, and S. A. Boardsen (2002), Small-scale field-aligned plasmaspheric density structures inferred from the Radio Plasma Imager on IMAGE, J. Geophys. Res., 107(A9), 1258, doi:10.1029/2001JA009199.

Chappell, C. R. (1974), Detached plasma regions in the magnetosphere, J. Geophys. Res., 79, 1861.

Chen, A. J., and R. A. Wolf (1972), Effects on the plasmasphere of a timevarying convfection electric field, Planet. Space Sci., 20, 483.

Craven, P. D., D. L. Gallagher, and R. H. Comfort (1997), Relative concentration of $\mathrm{He}^{+}$in the inner magnetosphere as observed by the DE 1 retarding ion mass spectrometer, J. Geophys. Res., 102, 2279.

Décréau, P. M. E. (1983), Fonctionnement d'une sonde quadripolaire sur satellite magnétosphérique (expérience GEOS); contribution à l'étude du comportement du plasma droid au voisinage de la plasmapause équatoriale, state doctorate thesis, Univ. de Orléans, Orléans, France.

Décréau, P. M. E., D. Carpenter, C. R. Chappell, R. H. Comfort, J. L. Green, R. C. Olsen, and J. H. Waite (1986), Latitudinal plasma distribu- 
tion in the dusk plasmaspheric bulge: Refilling phase and quasiequilibrium state, J. Geophys. Res., 91, 6929.

Fok, M.-C., J. U. Kozyra, A. F. Nagy, C. E. Rasmussen, and G. V. Khazanov (1993), A decay model of equatorial ring current and the associated aeronomical consequences, J. Geophys. Res., 98, 19,381.

Foster, J. C., P. J. Erickson, A. J. Coster, J. Goldstein, and F. J. Rich (2002), Ionospheric signatures of plasmaspheric tails, Geophys. Res. Lett., 29(13), 1623, doi:10.1029/2002GL015067.

Gallagher, D. L., P. D. Craven, and R. H. Comfort (2000), Global core plasma model, J. Geophys. Res., 105, 18,819.

Goldstein, J., R. W. Spiro, P. H. Reiff, R. A. Wolf, B. R. Sandel, J. W. Freeman, and R. L. Lambour (2002), IMF-driven overshielding electric field and the origin of the plasmaspheric shoulder of May 24, 2000, Geophys. Res. Lett., 29(16), 1819, doi:10.1029/2001GL014534.

Goldstein, J., M. Spasojević, P. H. Reiff, B. R. Sandel, W. T. Forrester, D. L. Gallagher, and B. W. Reinisch (2003), Identifying the plasmapause in IMAGE EUV data using IMAGE RPI in situ steep density gradients, J. Geophys. Res., 108(A4), 1147, doi:10.1029/2002JA009475.

Goldstein, J., B. R. Sandel, M. M. Thomsen, M. Spasojević, and P. H. Reiff (2004a), Simultaneous remote sensing and in situ observations of plasmaspheric drainage plumes, J. Geophys. Res., 109, A03202, doi:10.1029/ 2003JA010281.

Goldstein, J., B. R. Sandel, M. R. Hairston, and S. B. Mende (2004b), Plasmapause undulation of 17 April 2002, Geophys. Res. Lett., 31, L15801, doi:10.1029/2004GL019959.

Grebowsky, J. M. (1970), Model study of plasmaspheric motion, J. Geophys., Res., 75, 4329.

Green, J. L., B. R. Sandel, S. F. Fung, D. L. Gallagher, and B. W. Reinisch (2002), On the origin of kilometric continuum, J. Geophys. Res., 107(A7), 1105, doi:10.1029/2001JA000193.

Green, J. L., S. Boardsen, S. F. Fung, H. Matsumoto, K. Hashimoto, R. R. Anderson, B. R. Sandel, and B. W. Reinisch (2004), Association of kilometric continuum radiation with plasmaspheric structures, J. Geophys. Res., 109, A03203, doi:10.1029/2003JA010093.

Higel, B., and L. Wu (1984), Electron density and plasmapause characteristics at $6.6 R_{E}$ : A statistical study of the GEOS 2 relaxation sounder data, J. Geophys. Res., 89, 1583.

Khazanov, G. V., K. V. Gamayunov, and V. K. Jordanova (2003), Selfconsistent model of magnetospheric ring current and electromagnetic ion cyclotron waves: The 2-7, May 1998, storm, J. Geophys. Res., 108(A12), 1418, doi:10.1029/2003JA009833.

Lawrence, D. J., M. F. Thomsen, J. E. Borovsky, and D. J. McComas (1999), Measurements of early and late time plasmasphere refilling as observed from geosynchronous orbit, J. Geophys. Res., 104, 14,691.

Lemaire, J. F., and K. I. Gringauz (1998), The Earth's Plasmasphere, Cambridge Univ. Press, New York.

Liemohn, M. W., J. U. Kozyra, P. G. Richards, G. V. Khazanov, M. J. Buonsanto, and V. K. Jordanova (2000), Ring current heating of the thermal electrons at solar maximum, J. Geophys. Res., 105, 27,767.

Liemohn, M. W., A. J. Ridley, D. L. Gallagher, D. M. Ober, and J. U. Kozyra (2004), Dependence of plasmaspheric morphology on the electric field description during the recovery phase of the April 17, 2002 magnetic storm, J. Geophys. Res., 109, A03209, doi:10.1029/2003JA010304.

Lu, G., P. H. Reiff, M. R. Hairston, R. A. Heelis, and J. L. Karty (1989), Distribution of convection potential around the polar cap boundary as a function of the interplanetary magnetic field, J. Geophys. Res., 94, 13,447 .

Nishida, A. (1966), Formation of the plasmapause, or magnetospheric plasma knee, by the combined action of magnetosphere convection and plasma escape from the tail, J. Geophys. Res., 71, 5669.

Ober, D. M., J. L. Horwitz, and D. L. Gallagher (1997), Formation of density troughs embedded in the outer plasmasphere by subauroral ion drift events, J. Geophys. Res., 102, 14,595.

Park, C. G. (1973), Whistler observations of the depletion of the plasmasphere during a magnetospheric substorm, J. Geophys. Res., 78, 672.

Reinisch, B. W., et al. (2000), The radio plasma imager investigation on the IMAGE spacecraft, The radio plasma imager, Space Sci. Rev., 91, 319.

Reinisch, B. W., X. Huang, P. Song, J. L. Green, S. F. Fung, V. M. Vasyliunas, D. L. Gallagher, and B. R. Sandel (2004), Plasmaspheric mass loss and refilling as a result of a magnetic storm, J. Geophys. Res., 109, A01202, doi:10.1029/2003JA009948.

Ridley, A. J., T. I. Gombosi, and D. L. De Zeeuw (2004), Ionospheric control of the magnetosphere: Conductance, Ann. Geophys., 22, 567.

Sandel, B. R., et al. (2000), The extreme ultraviolet imager investigation for the IMAGE mission, Space Sci. Rev., 91, 197.

Sandel, B. R., R. A. Kind, W. T. Forrester, D. L. Gallagher, A. L. Broadfoot, and C. C. Curtis (2001), Initial results from the IMAGE extreme ultraviolet imager, Geophys. Res. Lett., 28, 1439.

Sandel, B. R., J. Goldstein, D. L. Gallagher, and M. Spasojevic (2003), Extreme ultraviolet imager observations of the structure and dynamics of the plasmasphere, Space Sci. Rev., 109, 25.

Song, X. T., C. Caudal, and R. Gendrin (1988), Refilling of the plasmasphere at the geostationary orbit: A Kp-dependent model deduced from GEOS-1 measurements of the cold plasma density, Adv. Space Res., 8 , 45

Spasojević, M., J. Goldstein, D. L. Carpenter, U. S. Inan, B. R. Sandel, M. B. Moldwin, and B. W. Reinisch (2003), Global response of the plasmasphere to a geomagnetic disturbance, J. Geophys. Res., 108(A9), 1340, doi:10.1029/2003JA009987.

Tobiska, W. K. (2004), SOLAR2000 irradiances for climate change, aeronomy, and space system engineering, Adv. Space Res., 34, 1736.

M. L. Adrian, NASA Goddard Space Flight Center, Greenbelt, MD 20771, USA.

D. L. Gallagher, NASA Marshall Space Flight Center, Huntsville, AL 35805, USA. (dennis.1.gallagher@nasa.gov)

M. W. Liemohn, Space Physics Research Laboratory, University of Michigan, Ann Arbor, MI 48109, USA. 